Canadian

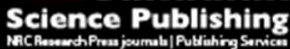

Canadian Journal of Microbiology Revue canadienne de de microbiologie

\title{
Comparative analysis of sigma factors RpoS, FliA and RpoN in Edwardsiella tarda
}

\begin{tabular}{|r|l|}
\hline Journal: & Canadian Journal of Microbiology \\
\hline Manuscript ID & cjm-2016-0158.R1 \\
\hline Manuscript Type: & Article \\
\hline Complete List of Authors: & $\begin{array}{l}\text { Song, ShanShan; State Key Laboratory of Bioreactor Engineering, East } \\
\text { China University of Science and Technology } \\
\text { Xue, Yuanyuan; State Key Laboratory of Bioreactor Engineering, East China } \\
\text { University of Science and Technology } \\
\text { Liu, Enfu; State Key Laboratory of Bioreactor Engineering, East China } \\
\text { University of Science and Technology } \\
\text { Wang, Keping; State Key Laboratory of Bioreactor Engineering ,East China } \\
\text { University of Science and Technology, } \\
\text { Zhang, Yuanxing; State Key Laboratory of Bioreactor Engineering ,East } \\
\text { China University of Science and Technology, } \\
\text { Wu, Haizhen; State Key Laboratory of Bioreactor Engineering ,East China } \\
\text { University of Science and Technology, } \\
\text { Zhang, Huizhan; State Key Laboratory of Bioreactor Engineering, East } \\
\text { China University of Science and Technology }\end{array}$ \\
\hline Keyword: & \begin{tabular}{l} 
Edwardsiella tarda, Sigma factor, Comparative proteomic analysis \\
\hline
\end{tabular} \\
\hline
\end{tabular}




\section{Comparative analysis of sigma factors RpoS, FliA, and RpoN in}

\section{Edwardsiella tarda}

ShanShan Song, Yuanyuan Xue, Enfu Liu, Keping Wang, Yuanxing Zhang, Haizhen Wu*, Huizhan Zhang*

State Key Laboratory of Bioreactor Engineering, East China University of Science and Technology, Shanghai 200237, P. R. China

ShanShan Song E-mail: shss33@163.com

Yuanyuan Xue E-mail: xyymetal@126.com

Enfu Liu E-mail: 531117153@qq.com

Keping Wang E-mail: wangkeping68@126.com

Yuanxing Zhang E-mail: yxzhang@ ecust.edu.cn

*Corresponding Authors:

Haizhen $\mathrm{Wu}$

E-mail:wuhzh@ecust.edu.cn

Phone: +8621 64252507

Fax: +8621624252507

Huizhan Zhang

E-mail: huizhzh@ecust.edu.cn

Phone: +8621 64252507

Fax: +8621624252507 


\begin{abstract}
Sigma factors are important regulators that bacteria employ to cope with environmental changes. Studies on the functions of sigma factors have uncovered their roles in many important cellular activities, such as growth, stress tolerance, motility, biofilm formation, and virulence. However, comparative analyses of sigma factors, which examine their common and unique features or elucidate their cross-regulatory relationships, have rarely been conducted for Edwardsiella tarda. Here, we characterized and compared motility and resistance to oxidative stress of E. tarda strains complemented with $r p o S$, fliA, and rpoN mutants. The results suggest that the sigma factors, FliA and RpoN, regulated motility, whereas RpoS exhibited no such function. RpoS and RpoN were essential for oxidative stress resistance, whereas FliA had no obvious impact under oxidative stress conditions. Furthermore, 2-dimensional gel electrophoresis (2-DE) based proteomics analysis, combined with matrix-assisted laser desorption/ionization time-of-flight mass spectrometry (MALDI-TOF MS/MS), revealed 12 differentially expressed protein spots that represented 11 proteins between the mutant and wild-type strains. Quantification of the expression of target genes by quantitative reverse transcription PCR (qRT-PCR) confirmed the results of our proteomics analysis. Collectively, these results suggest that these sigma factors are multi-functional mediators involved in controlling the expression of many metabolic pathway genes.
\end{abstract}

Keywords Edwardsiella tarda, Sigma factor, Comparative proteomic analysis 


\section{Introduction}

Edwardsiella tarda, a member of the family Enterobacteriaceae, is a gram-negative bacterium and major aquaculture pathogen that infects a wide range of hosts, including marine and fresh-water fish species (Slaven et al. 2001). Infection by E. tarda results in an enterohemorrhagic septicemic disease (i.e. edwardsiellosis), which causes severe economic losses in the aquaculture industry (Mohanty and Sahoo 2007). In addition, as an opportunistic pathogen, E. tarda has developed a complex mechanism for intestinal and extra-intestinal infection in fish, animals, and human beings (Helmann and Chamberlin 1988; Nelson et al. 2009; Tsuji et al. 2008).

There are five alternative sigma factors in E. tarda, namely $\sigma^{24}($ rpoE $), \sigma^{32}(r p o H), \sigma^{38}($ rpoS $)$, $\sigma^{54}(r p o N)$, and $\sigma^{28}(f l i A)$, each of which is encoded by only one gene copy. These sigma factors are involved in maintaining cellular homeostasis in response to various environmental stressors. RpoS is key for the survival of many pathogens, and has been demonstrated to play an essential role in survival under acute food shortage conditions and in protection against acidic, osmotic, and oxidative stress (Landini et al. 2014; Schellhorn 2014). The flagellum mediates bacteria motility and is a potential factor in bacterial virulence. Therefore, FliA is a potential virulence factor due to its essential roles in flagellar filament structure and biofilm formation. RpoN was originally found in some enteric bacteria, such as Escherichia, but is widely distributed among the bacteria (Buck et al. 2000; Merrick and Coppard 1989). It was reported that RpoN plays important roles in nitrogen utilization (Riordan et al. 2010). In Escherichia coli, RpoN controls the expression of approximately 30 operons, half of which involved in nitrogen assimilation (Riordan et al. 2010). Moreover, RpoN is also responsible for virulence, stress responses (Gruber and Gross 2003), and flagellum biosynthesis in Xanthomonas campestris (Yang et al. 2009).

In our previous studies, an $r p o N$ deletion mutant was constructed from the E. tarda wild-type strain EIB202 and investigated in comparison to the wild-type strain (Wang et al. 2012). 
RpoN plays an important role in the control of virulence genes and is essential for resistance to $\mathrm{H}_{2} \mathrm{O}_{2}$, starvation, high osmotic pressure, and acid (Wang et al. 2012). In this study, we constructed rpoS and fliA deletion mutants and complemented strains. Through a set of physiology experiments, we characterized and compared motility and resistance to oxidative stress for these E. tarda strains. Our results suggested that FliA and RpoN regulated motility, whereas RpoS had no such function. RpoS was essential for resistance to oxidative stress, whereas FliA had no obvious impact. Furthermore, 2-DE based proteomics approaches were applied to investigate differentially expressed proteins among the E. tarda strains, using the whole-cell protein fraction of E. tarda EIB202, rpoS, and fliA and rpoN deletion mutants. Comparative proteomic analysis, combined with MALDI-TOF MS/MS, revealed 12 identified protein spots that represented 11 proteins between the mutant strains and the wild-type strain. These results support the conclusion that RpoS, FliA, and RpoN are multi-functional mediators involved in regulating the expression of various metabolic pathway genes. 


\section{Materials and methods}

\section{Bacterial strains, plasmids, and culture conditions}

Strains and plasmids used in this study are listed in Table S1. E. tarda strains were grown in TSB (tryptone, $15 \mathrm{~g} \mathrm{~L}^{-1}$; soya peptone, $5 \mathrm{~g} \mathrm{~L}^{-1}$; NaCl, $5 \mathrm{~g} \mathrm{~L}^{-1}$ ) or TSA (tryptone, $15 \mathrm{~g} \mathrm{~L}^{-1}$; soya peptone, $5 \mathrm{~g} \mathrm{~L}^{-1} ; \mathrm{NaCl}, 5 \mathrm{~g} \mathrm{~L}^{-1}$; agar, $\left.18 \mathrm{~g} \mathrm{~L}^{-1}\right)$ at $28{ }^{\circ} \mathrm{C}$. When necessary, an appropriate amount of antibiotics (Sigma-Aldrich, St. Louis, MO) were supplemented to the medium at the following final concentrations: $15 \mu \mathrm{g} \mathrm{mL}{ }^{-1}$ colistin $(\mathrm{Col}), 30 \mu \mathrm{g} \mathrm{mL} L^{-1}$ chloramphenicol (Cm), $50 \mu \mathrm{g} \mathrm{mL}^{-1}$ ampicillin (Amp), and $50 \mu \mathrm{g} \mathrm{mL}{ }^{-1}$ kanamycin (Kan). E. coli strains were grown in $\mathrm{LB}$ (tryptone, $10 \mathrm{~g} \mathrm{~L}^{-1}$; yeast extract, $5 \mathrm{~g} \mathrm{~L}^{-1} ; \mathrm{NaCl}, 10 \mathrm{~g} \mathrm{~L}^{-1}$ ) at $37^{\circ} \mathrm{C}$. Plasmids used in this study were constructed in E. coli strains and then transferred into E. tarda strains through electroporation or mating with E. coli SM10 $\lambda$ pir.

\section{Construction of $r p o S$ and $f l i A$ in-frame deletion mutants and complemented strains}

Primers used in this study are listed in Table S2. PCR amplified DNA fragments used for constructing the in-frame deletion mutations of $r p o S$ and fliA were generated respectively by overlap PCR. Two PCR fragments were obtained from E. tarda EIB202 genomic DNA with the primer pairs of rpoSU-H1-F plus rpoSU-H2-R (a 596-bp fragment containing the upstream segment of rpoS) and rpoSD-H1-F plus rpoSD-H2-R (a 833-bp fragment containing the downstream segment of $r p o S$ ) using Pfu DNA polymerase (Fermentas, Vilnius, Lithuania). A 1421-bp product was obtained by overlap PCR with the primers rpoSU-H1-F and rpoSD-H2-R. The overlap fragment containing a deletion from nucleotides 1-977 was sequenced and ligated into the suicide vector, pRE112, with $S a c \mathrm{I}$ and $K p n \mathrm{I}$ restriction sites. The resulting plasmid, pRED-rpoS, was mated from E. coli SM10 גpir into E. tarda EIB202. The markerless in-frame deletion rpoS mutant in EIB202 was constructed using a double-crossover markerless gene deletion system (Lan et al. 2007). A similar method was used to obtain the $\triangle$ fliA mutant. The selected mutants were verified by PCR and DNA sequencing. 
To construct the complemented strains, rpoS $S^{+}$and $f l i A^{+}$, an intact $r p o S$ and fliA gene containing the putative promoter region was amplified with primers RpoS-c-sen/RpoS-c-ant and FliAF-c-P1/FliA-c-P2, and then cloned into the HindIII-/BamHI-digested low-copy plasmid, pACYC184, to create pACYC-rpoS and pACYC-fliA. The plasmids were respectively transformed into the rpoS and fliA mutants by electroporation. The desired complemented strains were screened and verified by PCR.

\section{Motility assay}

E. $\operatorname{tard} a$ wild-type (wt) and mutant strains were streaked out on TSA-medium plates and incubated at $28{ }^{\circ} \mathrm{C}$ for $24 \mathrm{~h}$. For the cell motility test, a single colony of each strain was spotted onto a TSA-medium plate $(0.4 \%$ agar $)$ and incubated for $48 \mathrm{~h}$ at $28^{\circ} \mathrm{C}$.

\section{Physiological experiments}

Growth of E. tarda strains was measured in LB at $28{ }^{\circ} \mathrm{C}$. The oxidative challenges $(3 \%$ and $1.5 \% \mathrm{H}_{2} \mathrm{O}_{2}$ ) were carried out as described previously (Tian et al. 2008; Xiao et al. 2009) with some modification. E. tarda wt, $\Delta f l i A$, fli $A^{+}$, as well as the $\Delta r p o S$ and $r p o S^{+}$strains were evenly streaked on LB media and incubated at $25^{\circ} \mathrm{C}$ for $2 \mathrm{~h}$, then treated with $10 \mu \mathrm{L}$ of either $3 \%$ or $1.5 \% \mathrm{H}_{2} \mathrm{O}_{2}$, which was spotted on the agar with $6 \mathrm{~mm}$ sterile filter paper. The inhibition zones were measured after culturing for $48 \mathrm{~h}$.

\section{Preparation of soluble whole-cell extracts}

The protocol for protein extraction from E. tarda was based on the method described previously with modification (Daware et al. 2012). E. tarda strains were grown in DEME (KCcell, Beijing, China) for $24 \mathrm{~h}$. Cells were adjusted to an OD value of 0.5 at $600 \mathrm{~nm}$ using fresh DEME and inoculated into fresh DEME at 1:50 dilutions. Protein samples were isolated after 24 hours. Bacterial cultures were disrupted with a micro-sonicator on ice. TCA was added to precipitate proteins at a final concentration of $10 \%$, and the mixture was kept for 1 hour in an ice bath. The protein was collected by centrifugation at $15,000 \times g$ for $5 \mathrm{~min}$ at 
$4{ }^{\circ} \mathrm{C}$, followed by three washes with ice-cold acetone. The pellet was air dried and dissolved with lysis buffer (9 M urea, 2\% CHAPS, 1\% DTT, 1\% ampholyte 3-10 NL). Insoluble materials were removed by centrifugation at $15,000 \times g$ for $10 \mathrm{~min}$ at room temperature. The concentration of each protein sample was determined by the Bradford method (Bradford 1976).

\section{DIGE}

Experimental samples were compared in one experiment using 2D DIGE (Ettan DIGE, GE Healthcare, UK). All proteins were solubilized to a final concentration of $5 \mathrm{mg} / \mathrm{mL}$ with DIGE lysis buffer (30 mM Tris, $7 \mathrm{M}$ urea, $2 \mathrm{M}$ thiourea, 4\% CHAPS). The $\mathrm{pH}$ of protein lysates was adjusted to $\mathrm{pH} 8.5$ with $50 \mathrm{mM} \mathrm{NaOH}$. A pool, to be used as an internal standard sample, was generated from an equal amount $(25 \mu \mathrm{g})$ of all 12 samples. CyDye was reconstituted in anhydrous DMF to a working solution of $0.4 \mathrm{mM}$. Protein samples were then labeled according to the standard protocol using the CyDye DIGE minimal labeling kit (GE Healthcare). In brief, $1 \mu \mathrm{L}$ of $\mathrm{Cy} 3$ or Cy5 dye DIGE fluors was added to the corresponding protein lysate (see Table S3) so that $50 \mu \mathrm{g}$ of each sample was labeled with $400 \mathrm{pmol}$ of fluor. Cy2 $(6 \mu \mathrm{L})$ was added to the internal standard protein lysate. The reactions were incubated on ice in the dark for thirty minutes and then terminated by adding $1 \mu \mathrm{L}$ of $10 \mathrm{mM}$ lysine (Sigma-Aldrich, St. Louis, MO) for every $400 \mathrm{pmol}$ of fluor. For each gel, $50 \mu \mathrm{g}$ of internal standard sample protein was mixed together with two experimental samples according to Table S3, and the volume was adjusted to $450 \mu \mathrm{L}$ with rehydration buffer (9 M Urea, $2 \%$ CHAPS, 1\% DTT, $1 \%$ pharmalyte $\mathrm{pH} 3-10 \mathrm{NL}$, trace bromophenol blue). The procedure for the separation of protein samples was the same as normal 2-DE, except that all the processes were performed in the dark. When the second dimension separation was finished, the gels were scanned using a Typhoon ${ }^{\mathrm{TM}}$ FLA 9000 biomolecular imager (GE Healthcare, Uppsala, Sweden).

\section{2-DE and silver staining}

The samples were separated using a $24 \mathrm{~cm}$ Immobiline DryStrip in the $\mathrm{pH}$ range of 3-10 NL 
according to the manufacturer's instructions (GE Healthcare, Uppsala, Sweden). First-dimension isoelectric focusing was performed with the Ettan ${ }^{\mathrm{TM}}$ IPGphor ${ }^{\mathrm{TM}}$ Isoelectric Focusing System (GE Healthcare, Uppsala, Sweden). Proteins were loaded by passive in-gel rehydration for 12 hours. IEF was performed under the following conditions: $100 \mathrm{~V}$ for $1 \mathrm{~h}$, $200 \mathrm{~V}$ for $1 \mathrm{~h}, 500 \mathrm{~V}$ for $1 \mathrm{~h}, 1,000 \mathrm{~V}$ for $1 \mathrm{~h}$, gradient $10,000 \mathrm{~V}$ for $3 \mathrm{~h}$, and a total focusing of $100 \mathrm{kVh}$ at $10,000 \mathrm{~V}$. After IEF, the IPG strips were equilibrated for $15 \mathrm{~min}$ in reducing equilibration buffer (6 M Urea, $75 \mathrm{mM}$ Tris- $\mathrm{HCl}, \mathrm{pH} 8.8,34.5 \%$ (v/v) Glycerol, 2\% (w/v) SDS, $1 \%(\mathrm{w} / \mathrm{v}) \mathrm{DDT})$ and subsequently alkylated for $15 \mathrm{~min}$ in alkylation equilibration buffer (6 M Urea, $75 \mathrm{mM}$ TrisHCl pH 8.8, 34.5\% (v/v) Glycerol, 2\% (w/v) SDS, 4\% (w/v) acrylamide (Sigma, St. Louis, USA)). The second dimension separation, SDS-PAGE, was performed in $11 \%$ polyacrylamide gels using an $\operatorname{Ettan}^{\mathrm{TM}}$ 2-D DIGE system (GE Healthcare, Uppsala, Sweden) in Tris-glycine buffer.

Silver staining was performed according to a previous report (Rao et al. 2004). The gels were fixed in $30 \%$ ethanol, $10 \%$ acetic acid for $30 \mathrm{~min}$, followed by two rinses in $20 \%$ ethanol for 10 minutes each and then two rinses in water for 10 minutes each. The gels were sensitized by soaking in $0.8 \mathrm{mM}$ sodium thiosulfate for one minute, followed by two rinses in water for one minute each. $12 \mathrm{mM}$ silver nitrate was used to impregnate the gel for 20 minutes, followed by two rinses in water for 30 seconds each. The gel was then transferred into the developer solution (2\% (w/v) sodium carbonate plus $400 \mu 137 \%$ methanal per liter). When bands appeared, the developer was replaced with stop solution $(4 \%(\mathrm{w} / \mathrm{v})$ Tris and $2 \%(\mathrm{v} / \mathrm{v})$ acetic acid), in which the gel was soaked for 30 minutes. After two 30 minute washes in water, the gel was scanned on a scanner (EPSON 11000XL, Nagano-ken, Japan).

\section{Protein identification by MALDI-TOF/TOF analysis}

The reproducible spots of interest were excised and identified by a plus 4800 matrix-assisted laser desorption/ionization time-of-flight/time-of-flight (MALDI-TOF/TOF) analyzer (Applied Biosystems, Foster City, CA, USA). The acquired MS spectra were submitted to the MASCOT search engine for protein/peptide sequence identification using the NCBI and 
MSDB protein databases. The parameters were as follows: fragment mass tolerance, $\pm 0.8 \mathrm{Da}$; one missed cleavage per peptide; fixed modifications of carbamidomethyl; peptide mass tolerance, $100 \mathrm{ppm}$; peptide charge state, 1+. A protein score of more than 45 was considered statistically significant, $\mathrm{p}<0.05$, (Perkins et al. 1999).

\section{RNA preparation, reverse transcription, and quantitative PCR}

Total RNA was extracted with the High Pure RNA Isolation Kit (Roche, Mannheim, Germany) following the recommendations of the manufacturer. cDNA was synthesized from $1 \mu \mathrm{g}$ of total RNA using reverse transcriptase (Roche, Mannheim, Germany) following the manufacturer's instructions and amplified by PCR. Quantitative PCR experiments were done in triplicate in a qPCR Bio-Rad CFX96 System using SYBR Premix (Takara Bio, Shiga, Japan). The relative expression of each gene was quantified by the Log2 RQ method (relative

quantity, $\mathrm{RQ}=2^{-\Delta \Delta \mathrm{CT}}$ ), using $r p o \mathrm{D}$ as an endogenous control. Sequences for the primers used are listed in Supplemental Table S2. 


\section{Results}

Construction of the rpoS and fliA deletion mutants and complemented strains of $E$. tarda EIB202

Using a double-crossover markerless gene deletion system with the suicide vector, pRE112, a 977-bp (from 1 to 977 bp) core portion of the 987-bp rpoS gene, and a 692-bp (from 26 to $717 \mathrm{bp}$ ) core portion of the 723-bp fliA gene were deleted in E. tarda EIB202. For each gene, three $\mathrm{Cm}$-sensitive $\left(\mathrm{Cm}^{\mathrm{s}}\right)$ second-crossover recombinants were selected as candidate gene deletion strains and confirmed by PCR, DNA sequencing, and Southern blot (Fig. S1 and Fig. S2). The $r p o S$ and fliA deletion mutants were named $\Delta r p o S$ and $\Delta f l i A$, respectively.

The full ORFs of rpoS and fliA were amplified by PCR and ligated into the plasmid, pACYC184, and the resulting complementation plasmids were named pACYC-rpoS and pACYC-fliA, which were subsequently introduced into $\Delta \mathrm{rpoS}$ and $\Delta \mathrm{fliA}$, respectively, and verified by PCR analysis and sequencing (data not shown). The resulting complementation strains were named $r p o S^{+}$and fliA $^{+}$.

\section{FliA, but not RpoS, is essential for motility of E. tarda}

To investigate the effect of sigma factors on motility, we compared motility among the $E$. tarda strains. E. tarda wt, $\Delta r p o S$, and $\Delta f l i A$ were inoculated vertically in $0.4 \%$ LB agar media. After being cultured for $24 \mathrm{~h}$, motility was fully lost for $\Delta f l i A$, but was restored when $\Delta f l i A$ was complemented with pACYC184-fliA (Fig. 1). Moreover, the motility of $\triangle f l i A$ was not enhanced after prolonged growth for $48 \mathrm{~h}$ (data not shown). This was also observed for $\Delta r p o N$ in our previous research (Wang et al. 2012). In contrast, the rpoS mutant showed normal motility, which was highly similar to the wild-type strain (Fig. 1). These results demonstrated that fliA and $r p o N$, but not $r p o S$, are important regulators that are required for motility in $E$. tarda. 


\section{RpoS, but not FliA, is essential for oxidative stress resistance}

In our previous work, we found that $r p o N$ was an essential regulatory gene for protection against $\mathrm{H}_{2} \mathrm{O}_{2}$ stress (Wang et al. 2012). Furthermore, an earlier report suggested that RpoS, a sigma factor involved in general stress response, was important for responding to oxidative stress by controlling the transcriptions of katB and $\operatorname{sodB}$ in E. tarda (Xiao et al. 2009). However, the role of fliA in oxidative stress is unknown. In order to evaluate the function of FliA in stress adaptation of $E$. tarda, we investigated the growth sensitivity of $E$. tarda strains when exposed to $\mathrm{H}_{2} \mathrm{O}_{2}$. E. tarda wt, $\Delta$ fliA and fliA $A^{+}, \Delta r p o S$ and $r p o S^{+}$, and $\Delta r p o N$ and $r p o N^{+}$ (positive control) were treated with different concentrations ( $3 \%$ or $1.5 \%$ ) of $\mathrm{H}_{2} \mathrm{O}_{2}$, and the inhibition zones were measured after culturing for 48 h. As shown in Fig. 2, $\Delta r p o S$ was about $50 \%$ less resistant to $\mathrm{H}_{2} \mathrm{O}_{2}$ than the wild-type was, whereas the rpoS complemented strain, $r p o S^{+}$, had a similar sensitivity to oxidative stress as that of the wild-type strain. Similarly, $\Delta r p o N$ was slightly more sensitive compared to wild-type, showing about a $16 \%$ decrease in growth in response to $\mathrm{H}_{2} \mathrm{O}_{2}$, whereas $r p o N^{+}$restored sensitivity to wild-type levels. These results are similar to our previous work (Wang et al. 2012). Conversely, the fliA deletion mutant did not exhibit the same sensitivity to oxidative stress as $\Delta r p o S$. These results indicate that RpoS and RpoN played important roles in oxidative stress resistance, whereas FliA had no such regulatory role.

\section{Identification of proteins differentially expressed amongst $E$. tarda wild-type, $\Delta r p o S$,} $\Delta f l i A$, and $\triangle r p o N$ strains

In order to establish an in-depth understanding of the function of the sigma factors in terms of mediating the levels of protein expression, we applied a comparative proteomic analysis to identify differentially expressed proteins among the wt, $\Delta r p o S, \Delta f l i A$, and $\Delta r p o N$ strains. Total soluble proteins were extracted from the four E. tarda strains, which were cultured in DEME media at $25^{\circ} \mathrm{C}$ for $24 \mathrm{~h}$. Soluble whole-cell protein extracts were then subjected to 2D-DIGE (Fig. 3A). A total of 723 unique spots were identified from all the images, and differences between wt/ $\operatorname{rpoS}$, wt/ $\Delta f l i A, \mathrm{wt} / \Delta r p o N, \Delta r p o S / \Delta f l i A, \Delta r p o S / \Delta r p o N$ and $\Delta f l i A / \Delta r p o N$ were 
analyzed with DeCyder software (GE Healthcare, Uppsala, Sweden) using default parameters. In parallel, the same protein samples were also subjected to 2-DE, stained with silver, and analyzed by imagemaster software (GE Healthcare, Uppsala, Sweden). Thirty protein spots that showed similar differential trends $(>1.1$ times, $\mathrm{p}<0.05)$ between $2 \mathrm{D}-\mathrm{DIGE}$ and 2-DE were selected and further analyzed by MALDI-TOF-MS/MS. Mass spectrometry analysis successfully identified 10 differentially expressed proteins (Table 1). Moreover, the same soluble whole-cell protein extracts were also resolved by 2-DE. Protein spots were visualized following silver staining (Fig. 3B). Two new differential proteins were further identified $(>2$-fold, $\mathrm{p}<0.05)$ (Table 1). The relative abundance levels of the 12 identified protein spots between the mutant strain and the wild-type strain are shown in Fig. 4 and Table S4, respectively.

Among the identified proteins spots, we found 4 spots involved in nitrogen metabolism, namely spots $4721,5708,6601$, and 2055 , corresponding to $\operatorname{gadB1}$, gadB2, and $\operatorname{gln} A$, which encode two glutamate decarboxylases and one glutamine synthetase, respectively. Interestingly, mass spectrometric analysis revealed that spots 4721 and 5708 represented the same protein, although they separated with an apparently different isoelectric point during 2D-DIGE. In addition, strain-dependent similarity in relative expression levels of spots 4721 and 5708 was observed (Fig. 4). Spots 4721 and 5708 expression were downregulated by 1.72- and 2.11-fold, respectively, in the rpoS deletion mutant, but were upregulated by 1.86and 2.12-fold, respectively, in the fliA deletion mutant and by 1.36- and 1.52-fold, respectively, in the rpoN deletion mutant (Fig. 4). Spot 6601 represented a glutamate decarboxylase encoded by $\operatorname{gadB2}$, whose expression was similar with the expression profile of gadB1dramatically downregulated by 16.67 -fold in $\triangle r p o S$ and upregulated by $2.19-$ and 1.14 -fold in $\Delta f l i A$ and $\Delta r p o N$ strains, respectively (Fig. 4). Furthermore, another important nitrogen assimilation protein, the glutamine synthetase encoded by $g \ln A$ (Spot ID 2055), was found to have no obvious expression difference in $\triangle r p o S$ and $\triangle f l i A$, but was notably downregulated by $78 \%$ in $\Delta r p o N$. These results indicate that these sigma factors are important nitrogen metabolism regulators. 
Notably, the only catalase/peroxidase (encoded by ETAE_0889, spot ID 1521), which is the vital enzyme in protecting the cell from oxidative damage, was identified by the comparative proteomic analysis (Table. 1). Expression of catalase/peroxidase was downregulated by 1.32-fold in $\Delta r p o S$, whereas no obvious difference was observed in $\Delta f l i A$ (1.11-fold) and it was slightly upregulated in $\Delta r p o N$ (1.23-fold) (Fig. 4). These results corroborate the findings presented in Fig. 2, and further demonstrate that RpoS is the essential factor responsible for the oxidative stress response.

Spot 2615 was the function-uncharacterized protein, ETAE_1939. When rpoS was absent, its protein abundance decreased over 2-fold $(\mathrm{p}<0.05)$ compared to wt, $\Delta f l i A$, and $\Delta r p o N$, whose rpoS was not deleted and between which there were no obvious differences in the protein's relative abundances (Fig. S3).

To further demonstrate that the transcription profiles of the genes that encode these identified proteins are differentially regulated, quantitative reverse transcription PCR (qRT-PCR) was performed to quantify the transcript levels of several genes ( $\operatorname{gadB1}, \operatorname{gadB2}$, evpH, and ETAE_0889) between the three mutant strains and the wild-type strain. As shown in Fig. 5, transcription of the two glutamate decarboxylase encoding genes, gadB1 and $\operatorname{gadB2}$, was respectively 2.0- and 3.2-fold downregulated in $\Delta r p o S$, 2.4- and 1.9-fold upregulated in $\Delta f l i A$, and 1.1- and 0.9-fold in $\Delta r p o N$. These results were similar to the 2-D gel results (Fig. 4 and Table S4). In addition, transcript levels of ETAE_0889 and evpH were investigated. Most of the qRT-PCR results were in accordance with protein expression, except that the expression of ETAE_0889 in $\triangle r p o S$ showed an opposite trend compared to the 2-D gel results. 


\section{Discussion}

In the genome of E. tarda, 6 sigma factor encoding genes were found, namely $r p o D, r p o S$, fliA, rpoN, rpoE, and rpoH. RpoD is known to be the primary housekeeping sigma factor and is essential to fundamental cellular activities, thus it exists in all known bacteria (Gruber and Bryant 1997). The other 5 are alternative sigma factors, and each one has specific functions and are involved in different regulatory profiles (Helmann and Chamberlin 1988). Typically, alternative sigma factors regulate the expression of genes involved in specific physiological or developmental processes (Helmann and Chamberlin 1988). The role of RpoS, RpoN, and FliA have been investigated recently in E. tarda (Liu et al. 2014; Wang et al. 2012; Xiao et al. 2009; $\mathrm{Xu}$ et al. 2014), while the other two alternative sigma factors, RpoH and RpoE, have been demonstrated to play an important role in heat shock response and extracytoplasmic stress, respectively, in E. coli (Ishihama 2000). In the present and our previous studies (Wang et al. 2012), we successfully constructed $\Delta r p o N, \Delta r p o S$, and $\Delta f l i A$ mutants, which allowed us to characterize and compare the function of these sigma factors simultaneously. In addition, we also attempted to construct rpoH and rpoE deletion strains, which were unsuccessful. However, an rpoE overexpression strain was successfully obtained, and some preliminary data has shown that the transcription of $\operatorname{deg} P$ and another 5 genes are significantly affected when $r p o E$ is overexpressed (data not shown). This indicates an important role for RpoE, which is currently under investigation.

Through physiology experiments, significant roles of the three sigma factors (RpoN, RpoS, and FliA) were elucidated and interplays among them could be concluded. The alternative sigma gene, fliA, encodes a flagellar sigma factor, which was initially found to direct transcription of flagellar genes in E. coli (Ishihama 2000; Ohnishi et al. 1990). Similarly, FliA was shown to play an important role in the formation of flagella in E. tarda (Xu et al. 2014). Here, the $\Delta f l i A$ mutant presented a flagellum-impaired phenotype, which suggests that FliA was the direct sigma factor controlling motility in E. tarda. The transcription level of fliA was 
reduced significantly in an rpoN mutant (Dong et al. 2011; Liu et al. 2014), indicating a positive regulation of RpoN on fliA transcription. RpoN was also reported to positively control the expression of other flagella-related genes (Zhao et al. 2010). The deletion of $r p o N$ and consequent loss of motility in E. tarda (Wang et al. 2012) could be explained by the mechanism of regulatory interactions among these sigma factors. Therefore, RpoN could be a crucial motility regulator. In E. coli, rpoS mutants were highly motile in comparison to wild-type (Dong and Schellhorn 2009; Makinoshima et al. 2003), and the transcription level of fliA was increased in the both rpoS mutants of E. coli (Dong et al. 2011) and E. tarda ((Dong et al. 2011; Liu et al. 2014), so it was surprising that $r p o S$ had no impact on motility in E. tarda in the present study. Notably, recent studies have revealed that the regulation of gene expression by RpoS and RpoN proceeds by a substantial antagonistic regulatory manner (Dong et al. 2011), and that the transcriptional level of fliA was decreased in a $r p o N$ plus $r p o S$ double deletion mutant (Liu et al. 2014), which indicated that the regulatory effect of RpoS on motility was weaker than RpoN in E. tarda. In the present study, it is possible that the weak effect of the single deletion of rpoS on E. tarda motility was not detectable. In general, a complex regulatory interaction of the three sigma factors, RpoN, RpoS, and FliA, was observed in E. tarda.

To further elucidate the roles of the sigma factors in this study, we conducted a comparative proteomic analysis to determine the differentially expressed proteins by comparing proteomic profiles among wt, $\Delta r p o S, \Delta f l i A$, and $\Delta r p o N$. Consequently, 11 unique proteins representing 12 protein spots were identified. Interestingly, GadB1 was represented by two spots, 4721 and 5708 , that had the same molecular mass but different isoelectric points. This was further validated because the expression tendency of the two spots was highly similar among the strains. These results indicated that at least two forms of GadB1 exist in E. tarda. Notably, expression of the two glutamate decarboxylase encoding genes, gadB1 and gadB2, was decreased in the $\Delta r p o S$ mutant but increased in the $\Delta f l i A$ and the $\Delta r p o N$ mutants, suggesting that glutamate decarboxylases expression was positively regulated by RpoS but negatively by RpoN and FliA. It is well known that RpoN is mainly responsible for transcription of nitrogen 
utilization genes (Reitzer and Schneider 2001; Riordan et al. 2010). However, the observation that RpoS and FliA regulate nitrogen related genes like RpoN is unexpected, which further indicates that the target genes of the sigma factors are interwoven. Moreover, there is another possible mechanism that may exist, such as cross-regulation among the sigma factors. Given that limited experimental data is currently available regarding the direct interaction between these sigma factors and the identification of genes that are affected upon their deletion, further investigation is necessary.

Understanding the virulence mechanisms of the pathogenic bacteria, E. tarda, is globally important. The relationship between sigma factors and virulence is being studied by our team. Here, using blast analysis, we found that gene homologues for the ETAE_1939 protein with unknown function (Spot ID 2615), which was identified through comparative proteomic analysis, might only exist in pathogenic bacteria, like Salmonella enterica, Serratia marcescens, and Cronobacter sakazakii. In addition, the abundance of ETAE_1939 was significantly decreased (Table S4 and Fig. S3) and transcription was 2.4-fold downregulated (data not shown) in $\Delta r p o S$. Given that RpoS played essential roles in the virulence network, the newly identified protein, ETAE_1939, may also be involved in the pathogenicity of $E$. tarda.

\section{Acknowledgements}

The authors thank Dr. Zhaolan Mo (Institute of Oceanology, Chinese Academy of Sciences, China) for kindly sending the strains and plasmids used for mutant construction in this study.

This work was supported by the National Special Fund for State Key Laboratory of Bioreactor Engineering. 


\section{References}

Bradford, M.M. 1976. A rapid and sensitive method for the quantitation of microgram quantities of protein utilizing the principle of protein-dye binding. Analytical biochemistry 72 : 248-254.

Buck, M., Gallegos, M.T., Studholme, D.J., Guo, Y., and Gralla, J.D. 2000. The bacterial enhancer-dependent sigma(54) $(\operatorname{sigma}(\mathrm{N}))$ transcription factor. Journal of bacteriology 182(15): 4129-4136.

Daware, V., Kesavan, S., Patil, R., Natu, A., Kumar, A., Kulkarni, M., and Gade, W. 2012. Effects of arsenite stress on growth and proteome of Klebsiella pneumoniae. Journal of biotechnology 158(1-2): 8-16. doi: 10.1016/j.jbiotec.2011.12.013.

Dong, T., and Schellhorn, H.E. 2009. Control of RpoS in global gene expression of Escherichia coli in minimal media. Molecular genetics and genomics : MGG 281(1): 19-33.

Dong, T., Yu, R., and Schellhorn, H. 2011. Antagonistic regulation of motility and transcriptome expression by RpoN and RpoS in Escherichia coli. Molecular microbiology 79(2): $375-386$.

Gruber, T.M., and Bryant, D.A. 1997. Molecular systematic studies of eubacteria, using sigma70-type sigma factors of group 1 and group 2. Journal of bacteriology 179(5): 1734-1747.

Gruber, T.M., and Gross, C.A. 2003. Multiple sigma subunits and the partitioning of bacterial transcription space. Annual review of microbiology 57: 441-466.

Helmann, J.D., and Chamberlin, M.J. 1988. Structure and function of bacterial sigma factors. Annual review of biochemistry 57: 839-872.

Ishihama, A. 2000. Functional modulation of Escherichia coli RNA polymerase. Annual review of microbiology 54: 499-518.

Lan, M.Z., Peng, X., Xiang, M.Y., Xia, Z.Y., Bo, W., Jie, L., Li, X.Y., and Jun, Z.P. 2007. Construction and characterization of a live, attenuated esrB mutant of Edwardsiella tarda and its potential as a vaccine against the haemorrhagic septicaemia in turbot, Scophthamus 
maximus (L.). Fish \& shellfish immunology 23(3): 521-530.

Landini, P., Egli, T., Wolf, J., and Lacour, S. 2014. Sigma S, a major player in the response to environmental stresses in Escherichia coli: role, regulation and mechanisms of promoter recognition. Environmental microbiology reports 6(1): 1-13.

Liu, E., Ye, J., Song, S., Wang, K., Zhang, Y., and Zhang, H. 2014. Impact of co-deficiency of RpoN and RpoS on stress tolerance, virulence and gene regulation in Edwardsiella tarda. Journal of basic microbiology 54(7): 678-687.

Makinoshima, H., Aizawa, S., Hayashi, H., Miki, T., Nishimura, A., and Ishihama, A. 2003. Growth phase-coupled alterations in cell structure and function of Escherichia coli. Journal of bacteriology 185(4): 1338-1345.

Merrick, M.J., and Coppard, J.R. 1989. Mutations in genes downstream of the rpoN gene (encoding sigma 54) of Klebsiella pneumoniae affect expression from sigma 54-dependent promoters. Molecular microbiology 3(12): 1765-1775.

Mohanty, B.R., and Sahoo, P.K. 2007. Edwardsiellosis in fish: a brief review. Journal of biosciences 32(7): 1331-1344.

Nelson, J.J., Nelson, C.A., and Carter, J.E. 2009. Extraintestinal manifestations of Edwardsiella tarda infection: a 10-year retrospective review. The Journal of the Louisiana State Medical Society : official organ of the Louisiana State Medical Society 161(2): 103-106. Ohnishi, K., Kutsukake, K., Suzuki, H., and Iino, T. 1990. Gene fliA encodes an alternative sigma factor specific for flagellar operons in Salmonella typhimurium. Molecular \& general genetics : MGG 221(2): 139-147.

Perkins, D.N., Pappin, D.J., Creasy, D.M., and Cottrell, J.S. 1999. Probability-based protein identification by searching sequence databases using mass spectrometry data. Electrophoresis 20(18): 3551-3567.

Rao, P.S., Yamada, Y., Tan, Y.P., and Leung, K.Y. 2004. Use of proteomics to identify novel virulence determinants that are required for Edwardsiella tarda pathogenesis. Molecular microbiology 53(2): 573-586.

Reitzer, L., and Schneider, B.L. 2001. Metabolic context and possible physiological themes of 
sigma(54)-dependent genes in Escherichia coli. Microbiology and molecular biology reviews : MMBR 65(3): 422-444, table of contents.

Riordan, J.T., Tietjen, J.A., Walsh, C.W., Gustafson, J.E., and Whittam, T.S. 2010. Inactivation of alternative sigma factor $54(\mathrm{RpoN})$ leads to increased acid resistance, and alters locus of enterocyte effacement (LEE) expression in Escherichia coli O157 : H7. Microbiology 156(Pt 3): 719-730.

Schellhorn, H.E. 2014. Elucidating the function of the RpoS regulon. Future microbiology 9(4): 497-507.

Slaven, E.M., Lopez, F.A., Hart, S.M., and Sanders, C.V. 2001. Myonecrosis caused by Edwardsiella tarda: a case report and case series of extraintestinal E. tarda infections. Clinical infectious diseases : an official publication of the Infectious Diseases Society of America 32(10): 1430-1433.

Tian, Y., Wang, Q., Liu, Q., Ma, Y., Cao, X., and Zhang, Y. 2008. Role of RpoS in stress survival, synthesis of extracellular autoinducer 2, and virulence in Vibrio alginolyticus. Archives of microbiology 190(5): 585-594.

Tsuji, A., Hirasawa, K., Arakuma, T., Izumi, K., Kobori, K., Sunohara, K., Yoshizawa, N., Watanabe, H., and Izumiya, H. 2008. A 12-year-old boy with acute gastroenteritis caused by Edwardsiella tarda O4:H4. Journal of infection and chemotherapy : official journal of the Japan Society of Chemotherapy 14(6): 433-435.

Wang, K., Liu, E., Song, S., Wang, X., Zhu, Y., Ye, J., and Zhang, H. 2012. Characterization of Edwardsiella tarda rpoN: roles in sigma(70) family regulation, growth, stress adaption and virulence toward fish. Archives of microbiology 194(6): 493-504.

Xiao, J., Wang, Q., Liu, Q., Xu, L., Wang, X., Wu, H., and Zhang, Y. 2009. Characterization of Edwardsiella tarda rpoS: effect on serum resistance, chondroitinase activity, biofilm formation, and autoinducer synthetases expression. Applied microbiology and biotechnology 83(1): 151-160.

Xu, T., Su, Y., Xu, Y., He, Y., Wang, B., Dong, X., Li, Y., and Zhang, X.H. 2014. Mutations of flagellar genes fliC12, fliA and flhDC of Edwardsiella tarda attenuated bacterial motility, 
biofilm formation and virulence to fish. Journal of applied microbiology 116(2): 236-244.

Yang, T.C., Leu, Y.W., Chang-Chien, H.C., and Hu, R.M. 2009. Flagellar biogenesis of Xanthomonas campestris requires the alternative sigma factors RpoN2 and FliA and is temporally regulated by FlhA, FlhB, and FlgM. Journal of bacteriology 191(7): 2266-2275.

Zhao, K., Liu, M., and Burgess, R.R. 2010. Promoter and regulon analysis of nitrogen assimilation factor, sigma54, reveal alternative strategy for E. coli MG1655 flagellar biosynthesis. Nucleic acids research 38(4): 1273-1283. 


\section{Legends}

Fig. 1 The motility ability of $E$. tarda wild-type and sigma mutant. The $E$. tarda strains were inoculated vertically in $0.4 \%$ LB agar media. Cultures were grown at $25^{\circ} \mathrm{C}$ for $24 \mathrm{~h}$.

Fig.2 Histogram of inhibition zones of the $E$. tarda strains by $\mathrm{H}_{2} \mathrm{O}_{2}$. The $E$. tarda strains were streaked on LB agar and incubated at $25^{\circ} \mathrm{C}$ for $2 \mathrm{~h}$. Then $10 \mu \mathrm{L}$ of the indicated concentrations of $\mathrm{H}_{2} \mathrm{O}_{2}$ were spotted on the agar with a $6 \mathrm{~mm}$ sterile filter paper. The inhibition zones were measured after $48 \mathrm{~h}$ incubation. Error bars indicate standard deviations from 3 independent biological replicates.

Fig. 3 Analysis of differential proteins of $E$. tarda strains using 2-DE based proteomics. 19 Images of 2D DIGE and 2DE. Arrows indicate the corresponding proteins spots indicated in Table 3 (see below) identified by MALDI-TOF-MS/MS. A, image of 2D DIGE of Gel 1 internal Standard; B, image of wt by silver staining.

Fig. 4 The relative abundance ratios of each identified protein spots between the $E$. tarda strains.

Fig. 5 qRT-PCR analysis of transcription profiles of the encoding genes of several differential proteins. RNAs were extracted from $E$. tarda wild-type and mutant strains cultured in DEME media at $25^{\circ} \mathrm{C}$ for $24 \mathrm{~h}$. Relative fold changes represent the level of expression in the each mutant strain compared to the level of expression in the wild-type. Error bars indicate standard deviations from 3 independent biological replicates. ND: not detected. 
Table 1 Annotations and MS information of the identified differential proteins

\begin{tabular}{|c|c|c|c|c|c|c|c|c|c|c|c|}
\hline $\begin{array}{l}\text { Spot } \\
\text { ID }\end{array}$ & Protein Description & $\begin{array}{l}\text { Gene } \\
\text { Name }\end{array}$ & gi in NCBI & Locus_tag & $\mathrm{Mr}$ & $\mathrm{pI}$ & $\begin{array}{l}\text { Protein } \\
\text { Score }\end{array}$ & $\begin{array}{l}\text { Protein } \\
\text { Score } \\
\text { C.I.\% }\end{array}$ & $\begin{array}{l}\text { Total } \\
\text { Ion } \\
\text { Score }\end{array}$ & $\begin{array}{l}\text { Total } \\
\text { Ion } \\
\text { C.I.\% }\end{array}$ & $\begin{array}{l}\text { Pep. } \\
\text { Count }\end{array}$ \\
\hline 6601 & glutamate decarboxylase & $\operatorname{gadB2}$ & gi|269140211 & ETAE_2868 & 52395 & 5.97 & 109 & 100 & 36 & 97.561 & 12 \\
\hline 1419 & GTP-binding protein & $y i h K$ & gi|269140833 & ETAE_3492 & 67298 & 5.18 & 151 & 100 & 107 & 100 & 10 \\
\hline 2055 & glutamine synthetase & $g \ln A$ & gi|269140834 & ETAE_3493 & 51877 & 5.15 & 129 & 100 & 66 & 99.998 & 11 \\
\hline 1894 & transcription elongation factor & nus $A$ & gi|269137762 & ETAE_0404 & 54855 & 4.57 & 353 & 100 & 220 & 100 & 19 \\
\hline 1036 & $\begin{array}{l}\text { type VI secretion system protein } \\
\text { EvpH }\end{array}$ & evpH & gi|269139781 & ETAE_2436 & 95833 & 5.49 & 384 & 100 & 223 & 100 & 24 \\
\hline 2556 & serine endoprotease & & gi|269140114 & ETAE_2771 & 47077 & 7.64 & 117 & 100 & 76 & 100 & 8 \\
\hline 4721 & glutamate decarboxylase & $\operatorname{gadB1}$ & gi|269138142 & ETAE_0786 & 64480.4 & 5.78 & 195 & 100 & 154 & 100 & 9 \\
\hline 5708 & glutamate decarboxylase & $\operatorname{gadB1}$ & gi|269138142 & ETAE_0786 & 64480.4 & 5.78 & 213 & 100 & 156 & 100 & 11 \\
\hline 5812 & outer membrane protein (porin) & & gi|269139173 & ETAE_1826 & 42331.2 & 4.68 & 305 & 100 & 245 & 100 & 11 \\
\hline
\end{tabular}




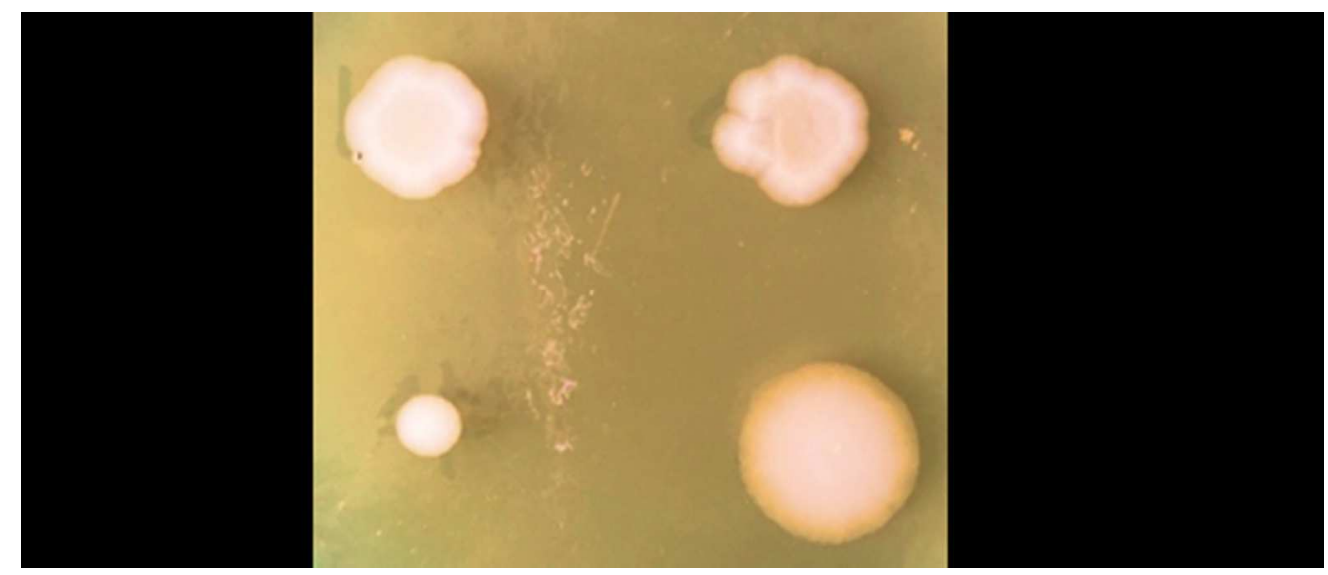

Fig. 1 The motility ability of E. tarda wild-type and sigma mutant. The E. tarda strains were inoculated vertically in $0.4 \% \mathrm{LB}$ agar media. Cultures were grown at $25^{\circ} \mathrm{C}$ for $24 \mathrm{~h}$. 


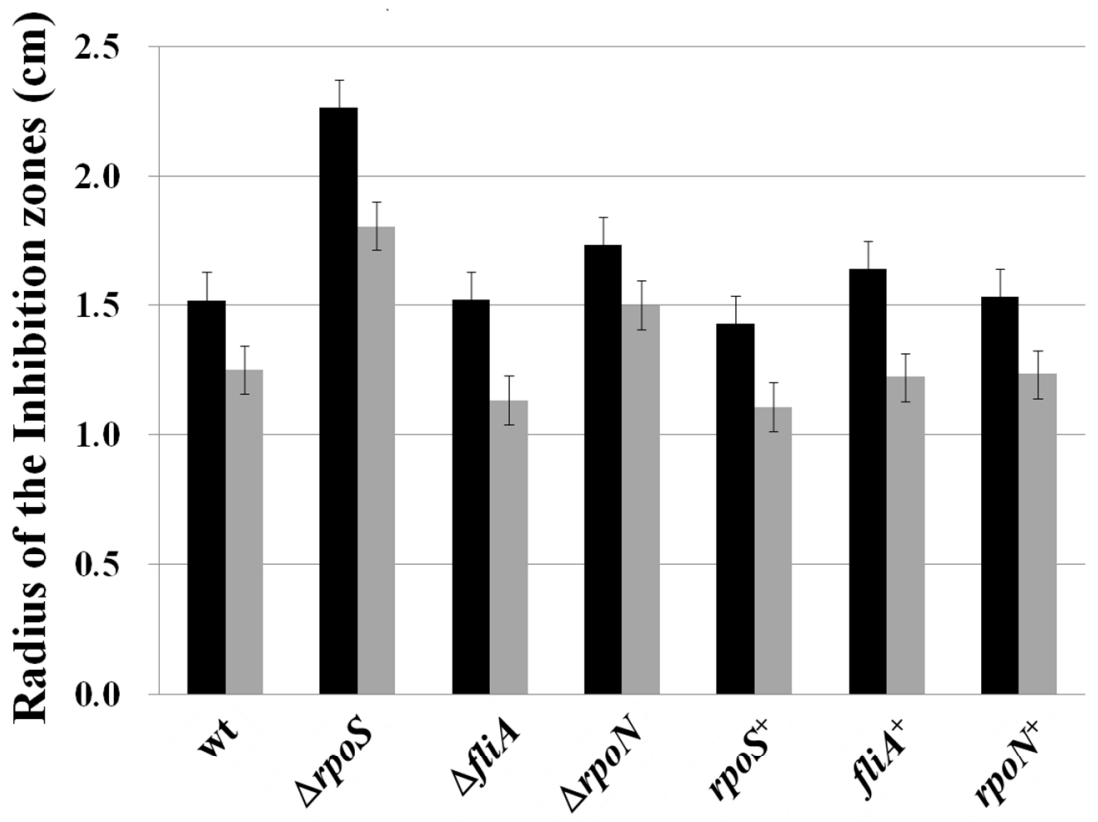

Fig. 2 Histogram of inhibition zones of the E. tarda strains by H2O2. The E. tarda strains were streaked on LB agar and incubated at $25^{\circ} \mathrm{C}$ for $2 \mathrm{~h}$. Then $10 \mu \mathrm{L}$ of the indicated concentrations of $\mathrm{H} 2 \mathrm{O} 2$ were spotted on the agar with a $6 \mathrm{~mm}$ sterile filter paper. The inhibition zones were measured after $48 \mathrm{~h}$ incubation. Error bars indicate standard deviations from 3 independent biological replicates.

$360 \times 249 \mathrm{~mm}(96 \times 96 \mathrm{DPI})$ 

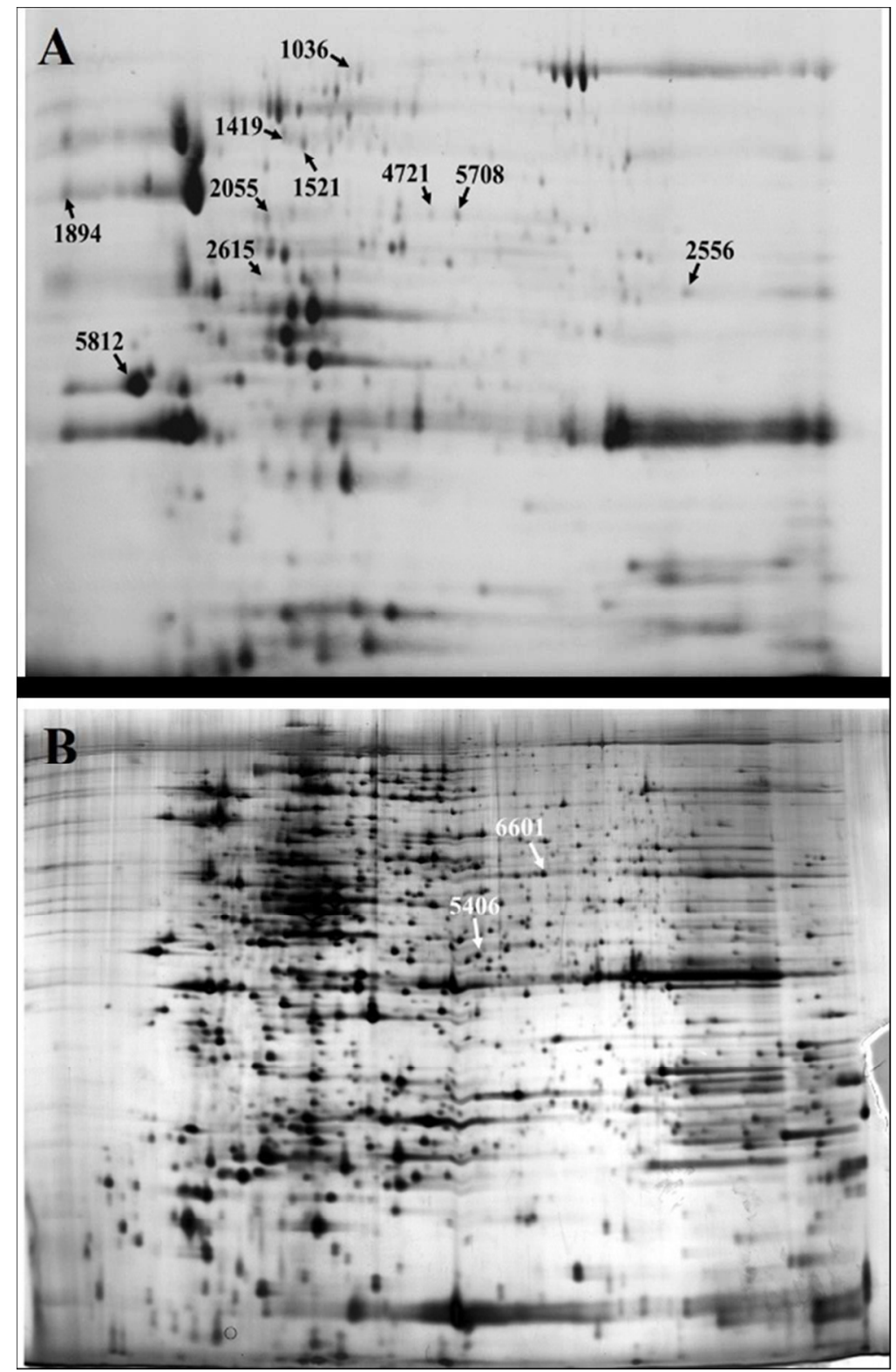

Fig. 3 Analysis of differential proteins of E. tarda strains using 2-DE based proteomics. Images of 2D DIGE and 2DE. Arrows indicate the corresponding proteins spots indicated in Table 1 (see below) identified by MALDI-TOF-MS/MS. A, image of 2D DIGE of Gel 1 internal Standard; B, image of wt by silver staining.

$$
133 \times 207 \mathrm{~mm}(150 \times 150 \mathrm{DPI})
$$




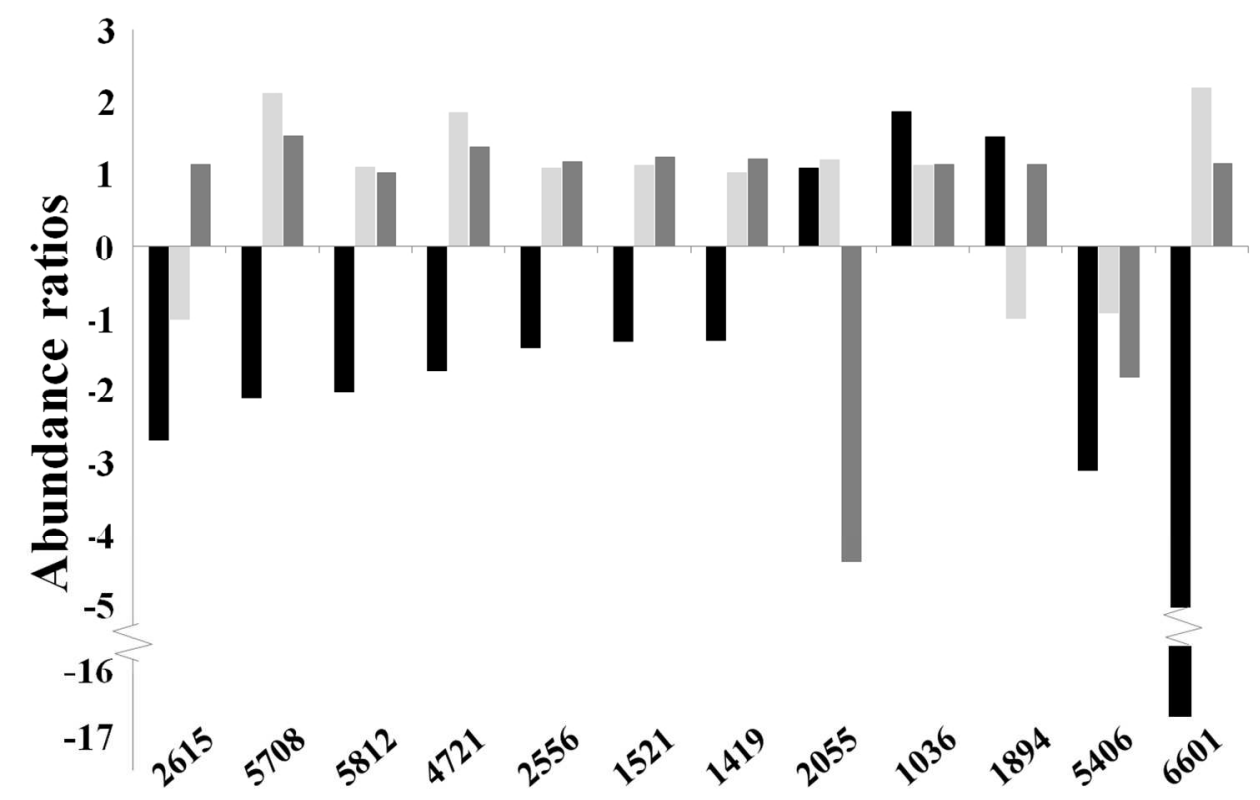

Fig. 4 The relative abundance ratios of each identified protein spots between the E. tarda strains. $379 \times 242 \mathrm{~mm}(96 \times 96 \mathrm{DPI})$ 


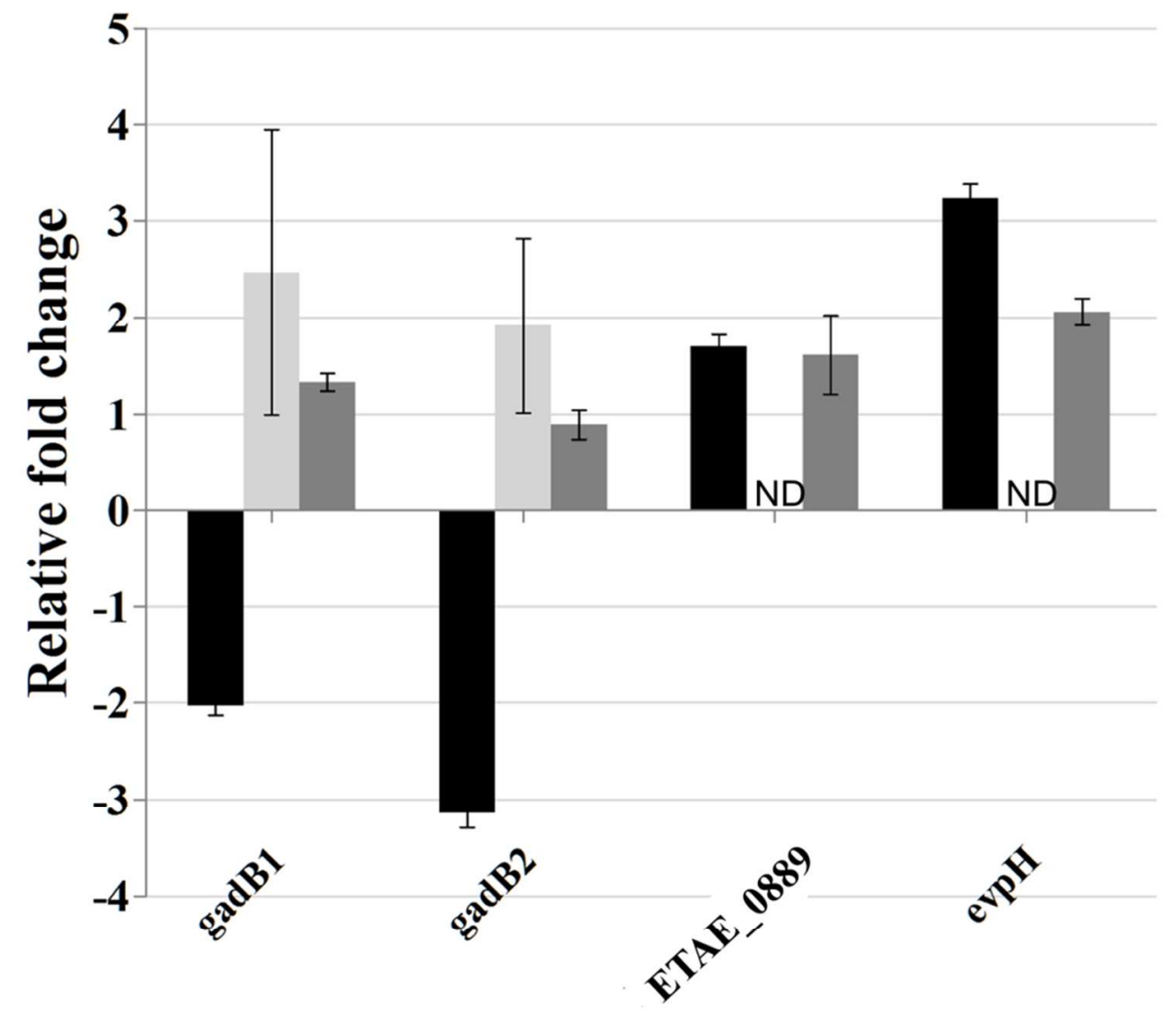

Fig. 5 qRT-PCR analysis of transcription profiles of the encoding genes of several differential proteins. RNAs were extracted from E. tarda wild-type and mutant strains cultured in DEME media at $25^{\circ} \mathrm{C}$ for $24 \mathrm{~h}$. Relative fold changes represent the level of expression in the each mutant strain compared to the level of expression in the wild-type. Error bars indicate standard deviations from 3 independent biological replicates. ND: not detected.

$289 \times 263 \mathrm{~mm}(96 \times 96 \mathrm{DPI})$ 